\title{
Recurrent Stye can be stopped by refractive correction - A case report
}

\author{
Author \\ Goutam Datta \\ Associate Professor, Department of Optometry, Tripura Institute of Paramedical Sciences, Agartala, \\ Tripura, India
}

\begin{abstract}
Recurrent Stye hampered educational performance in young adults. A case report of 21 years male college student was suffering around 3 years by recurrent Stye, which was treated several times by ocular medications temporarily. By investigation with objective and subjective refraction method the patient was corrected by minor sphero-cylindrical lens, which stopped the recurrent Stye permanently.

Keywords: Recurrent Stye, Sphero-cylindrical lens.
\end{abstract}

\section{Introduction}

External Hordeolum or Stye is an acute inflammation of the follicle of eyelash which is associated with Zeis or Moll gland. Usually Staphylococcus aureus are the causative organism of the inflammation ${ }^{[3]}$. Children and young adults are more sufferers, but may be occur at any age. Acute pain, swelling and heaviness of lid margin with discharge are the common symptoms of Stye $^{[1]}$. Redness and oedema will raise local temperature of the affected lid. A whitish round pus point grows at the lid margin. Hot compress with local antibiotic eye drops and ointments are the baseline treatment for Stye ${ }^{[3]}$. In severe case horizontal incision is necessary for drain out the pus.

\section{Case Report}

A 21 year old male college student was attend to the clinic with the complaints of lid swelling and acute eye ache in the right eye, when his terminal examination schedules are going on. $\mathrm{He}$ also reported that before his examination or in the time of examination he always suffering such type of eye related diseases since around 3 years in alternate eye, which hampered his examination preparation every time. Sometimes it was treated by hot compress or antibiotic eye drops. He also tested his blood sugar level around 7 months ago (fasting and postprandial) which was the normal in range.

He also reported that sometimes he suffered of fever at night time in the previous day. Due to acute pain, lid swelling with tenderness in right eye, it was unable to read vision chart in the effected eye on that moment. By the slit lamp examination it was found that whitish pus point were on the lid margin. The students was advised to apply hot compress 3 to 4 times with ciprofloxacin $(0.3 \%)$ local eye drops 4 times daily and chloramphenicol $(0.5 \%)$ eye ointments at bed times for 7 days in the affected eye. For reducing inflammation and acute pain he was advised for diclofenac sodium $(50 \mathrm{mg}$ ) tablet twice daily for 3 days. And was instructed him to attend for next follow up after 10 days (figure 1). 


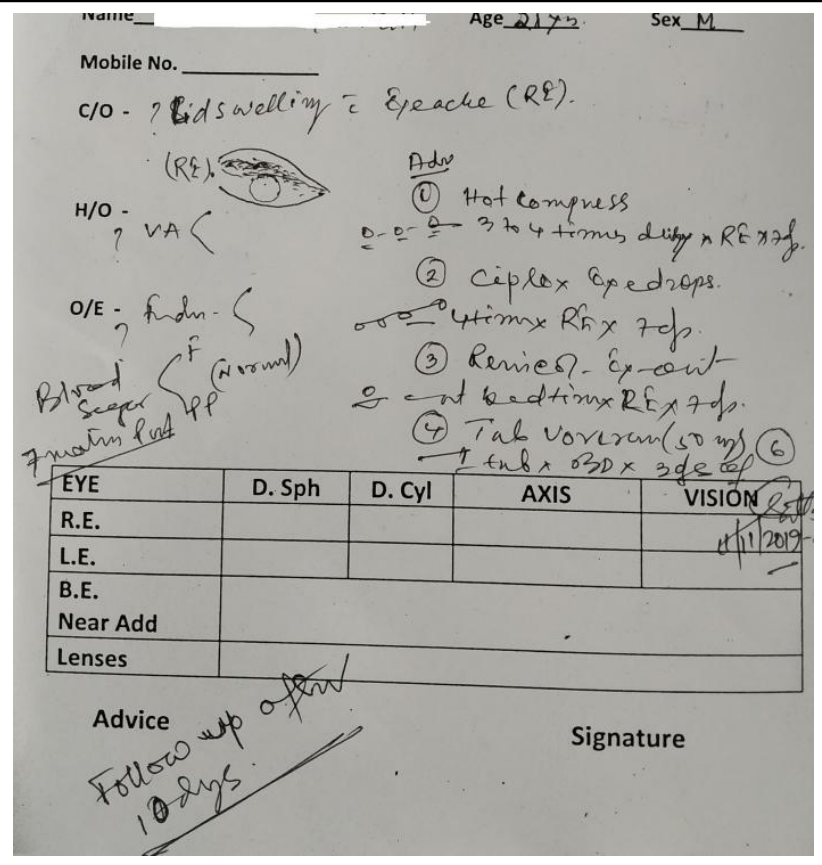

Fig. 1 First day prescription

As per instruction the college student was attend in the clinic after 12 days on his convenient time. $\mathrm{He}$ was completely relief from the external hordeolum. On routine clinical examination by slit lamp anterior segment including lid margin was normal. His distance visual acuity was $6 / 6(\mathrm{P})$ and near visual acuity from $33 \mathrm{~cm}$ distance N6 both eye respectively (figure 3). By Direct Ophthalmoscopy both fundus were observed healthy. In objective refraction by autorefractometer both eyes had mild spherocylindrical corrections (figure 2). Subsequently, in subjective refraction done by "Trial and Error" method and the spherical correction confirmed by "Fogging" method, followed by cylindrical correction by "Fun and block" method. His refractive correction was for right eye -0.25 Dsph with -0.25 Dcyl $\mathrm{X} 160^{\circ}$ and for left eye $0.25 \mathrm{Dsph}$ with $-0.25 \mathrm{Dcyl} \mathrm{X} 40^{\circ}$ (figure 3 ). After correction the distance visual acuity as well as near visual acuity in both eye was $6 / 6$ and N6 respectively. He was advised to wear spectacle regularly for 24hours, except sleeping and bathing time.

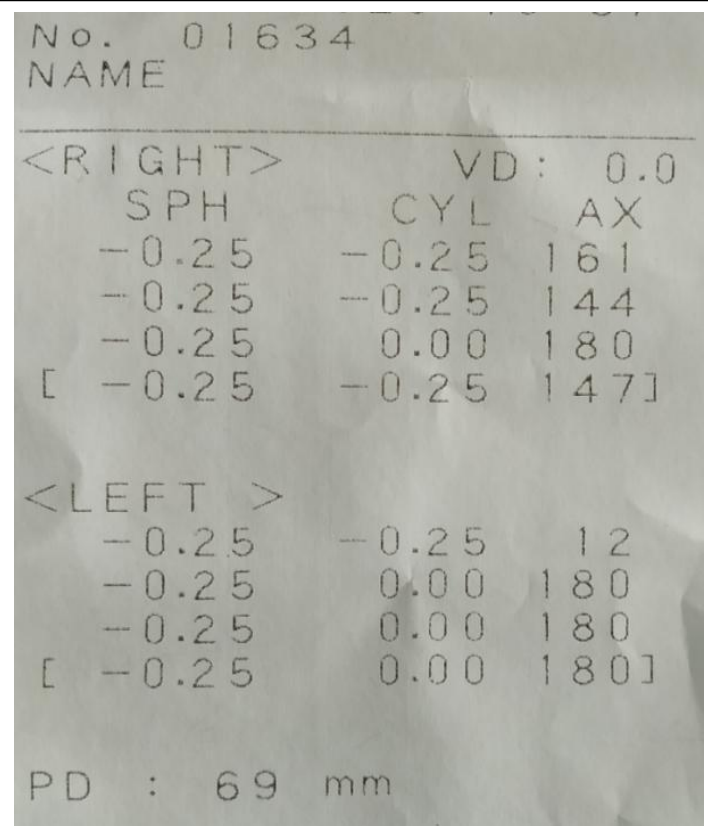

Fig. 2 Auto refractometer reading

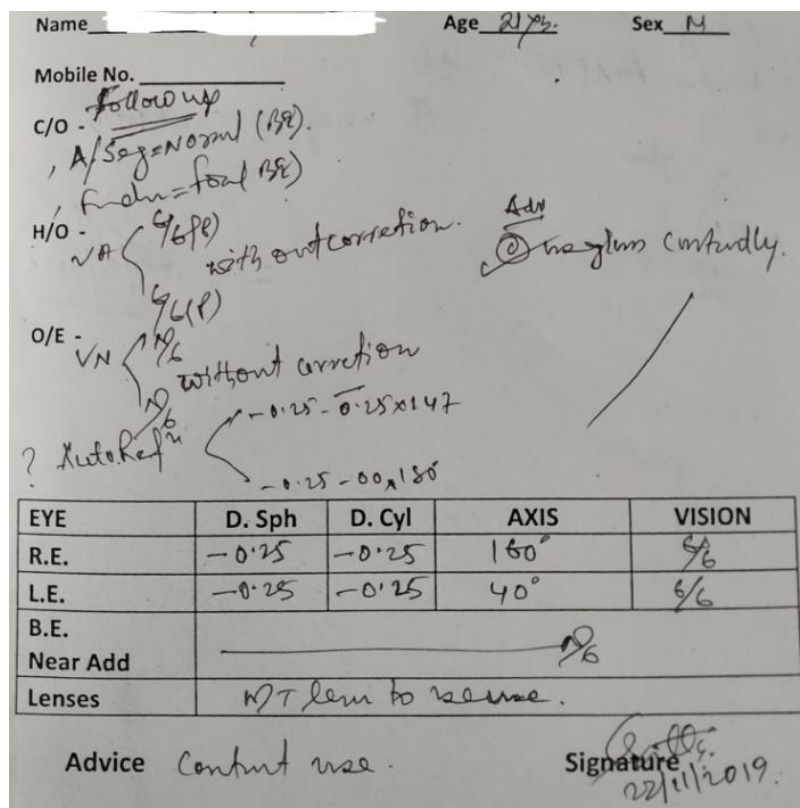

Fig. 3 Subjective refraction report

$3 \frac{1}{2}$ months letter the college student was came to the clinic wearing the spectacle, for further check up. He also reported that another college examination was completed without any ocular trouble. On that day the student was re-examined by the slit lamp for anterior segment, fundus by direct ophthalmoscope, distance visual acuity assessment and near visual acuity assessment with existing correction. Fundus and anterior segment were normal in both eyes. The visual acuity both distance and near are 6/6 and N6 with existing spectacle in both eyes respectively (figure 4). 


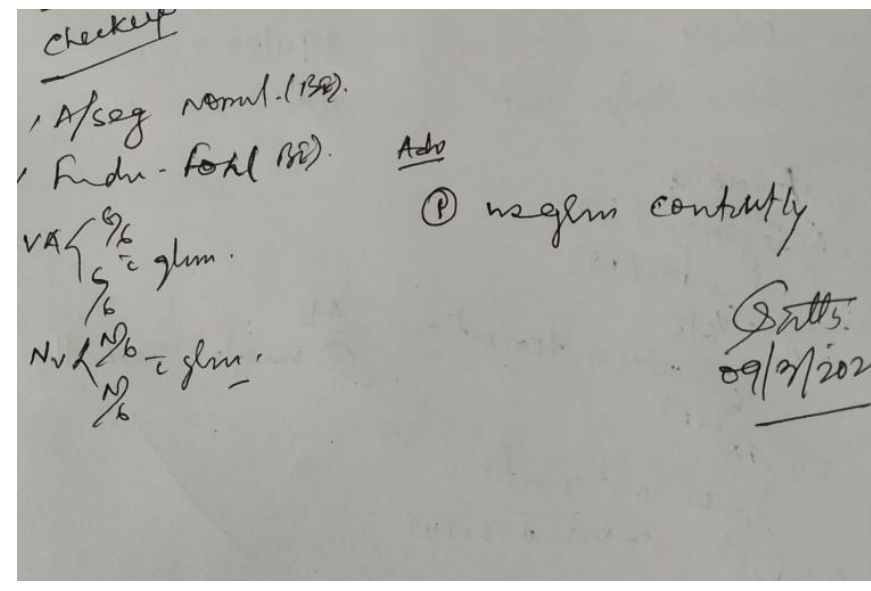

Fig. 4 Findings of $3 \frac{1}{2} 2$ months letter

\section{Discussion}

Uncorrected refractive errors are the second major causes of blindness in India. Neglect of refractive errors correction produces recurrent Stye. If a patient detect with Stye, medicine is the first preference to relieve from the Stye. Once the patient relieved from his trouble he never attend for further follow up examinations unless he attack next time by Stye ${ }^{[2]}$.

Normally when the eyes focus on a near object, the eyes converge to fix on the object without any stress. If the patients have refractive error, the patient will give in excessive effort to get clear vision. Over work on the concerned muscles, blood supply increased in conjunctiva, eyelids and the lid margins, which creates irritation, itchiness and sandy feeling in the eyes ${ }^{[2]}$. The patient will rub their eyes frequently; as a result the inflammation creates in the surroundings of lid margin, which will make the blockage of ducts of Zeis and Moll gland that produces Stye. Only minor refractive errors correction can overcome the problem. Stye is also associated with astigmatic correction, because the accommodative effort to clarify indistinct vision successfully ${ }^{[2]}$.

\section{Conclusions}

Uncorrected refractive errors do not always produce Stye; it is the predisposing factors of Stye. Refractive errors left uncorrected long time there is a possibility of recurrent Stye. It is true that in every case of minor astigmatic error need not have Stye. But there is a chance of many cases of Stye having minor astigmatic correction. The patient relieved from recurrent Stye by the correction of minor sphero-cylindrical lens for regular use and as well as he is performing his near work properly without any ocular stress.

\section{References}

1. S. K. Basak, Essentials of Ophthamology, 5th ed., p 127-28, Jan. 2015.

2. C. M. Sarma, "Styes and refractive erros their aetiological relationship," Indian journal of Ophthalmology., vol. 18, issue 2 pp. 56-58, 1970

3. G. Datta, Essentials of Paediatric Optometry, $1^{\text {st }}$ ed., p 182-84, Mar. 2018. 